\title{
El Trabajo de Campo en la formación integral del Profesor de Geografía: Una teorización fenomenológica desde la experiencia de los estudiantes
}

\author{
Field Work in the integral Training of the Geography Teacher: A \\ phenomenological theorization from the students' experience
}

\author{
Franklin Núñez Ravelo \\ franklingeove@hotmail.com \\ María Ugas Pérez \\ mariaeleneaugas_88@hotmail.com
}

\author{
Rossany Calderón Castellanos \\ marietcalderon19@gmail.com
}

Ferney Rivas Meriño

fernneyrivas@gmail.com

Universidad Pedagógica Experimental Libertador- Instituto Pedagógico de Caracas, Venezuela

Recibido octubre 2019 | Revisado nov - dic 2019 | Publicado 01 de enero 2020

\section{RESUMEN}

El estudio se ejecutó en el marco del proyecto desarrollado en la línea "Didáctica de la Geografía" del Centro de Investigaciones Geohistóricas del Instituto Pedagógico de Caracas (IPC), tuvo como premisa teleológica: develar con base en las experiencias-vivencias de 11 estudiantes del profesorado de Geografía e Historia. De allí que se asumió como ruta metodológica el paradigma interpretativo, desde el enfoque fenomenológico, basado en un diseño emergente sustentado en los momentos del método fenomenológico propuesto por Husserl (1900, 1913, 1929 y 1970), que orientó la sistematización de las experiencias de los informantes recabadas mediante entrevistas a profundidad. Se asume a modo de conclusión que el trabajo de campo permite empoderar al estudiante en su perfil profesional específico, toda vez que: propicia la autoconfianza y el crecimiento personal, lo vincula con el contexto en estudio generando sensibilidad y sentido de identidad profesional, le proyecta en el abordaje didáctico de situaciones específicas de realidades particulares aproximándolo al binomio escuela-comunidad, favorece la valoración de la estrategia por su aplicabilidad en distintos ámbitos, y potencia la búsqueda de soluciones a los problemas locales de uso del espacio y explotación indebida de los recursos, como vía para promover ciudadanos ambientalmente responsables desde el accionar pedagógico, individual y colectivo.

Palabras clave: campo de producción geográfica; educación; enseñanza de la geografía; formación de docentes; identidad docente; trabajo de campo

\begin{abstract}
The study was carried out within the framework of the project developed in the line "Didactics of Geography" of the Center for Geohistoric Research of the Pedagogical Institute of Caracas (IPC), had as a teleological premise: to reveal based on the experiences-experiences of 11 students of the Faculty of Geography and History. Hence, the interpretive paradigm was assumed as a methodological route, from the phenomenological approach, based on an emerging design based on the moments of the phenomenological method proposed by Husserl (1900, 1913, 1929 and 1970), which guided the systematization of the experiences of the informants collected through in-depth interviews. It is assumed by way of conclusion that the fieldwork allows to empower the student in their specific professional profile, since: it fosters selfconfidence and personal growth, links it with the context in study generating sensitivity and sense of professional identity, projects in the didactic approach of specific situations of particular realities approaching the school-community binomial, it favors the valuation of the strategy for its applicability in different areas, and promotes the search for solutions to local problems of space use and improper exploitation of resources, as a way to promote environmentally responsible citizens from the pedagogical, individual and collective actions.
\end{abstract}

Key words: geographical production field; education; Geography education; teaching identity; teacher training; field work 


\section{INTRODUCCIÓN}

En el acto didáctico, los docentes y estudiantes constituyen sujetos de inestimable relevancia, toda vez que son los protagonistas esenciales en la categoría enseñanza-aprendizaje. Esto inclusive, en aquellos modelos insertos en la educación no formal e informal, ya que estos demandan de una figura docente capaz de planificar la mediación didáctica, y con esta, las estrategias y actividades a fin de garantizar a los sujetos que aprenden, una interacción de manera protagónica en un franco proceso de construcción de los saberes.

Ahora bien, considerando que el mundo, no solo en su acepción planetaria, sino incluso personal, se percibe cada vez más complejo, demandando de los espacios universitarios investigativos, mayores niveles de transdiciplinariedad en el abordaje de situaciones contextuales específicas, aunado al hecho de exigir, la formación de docentes con competencias que les permitan ser coparticipe en las comunidades en dónde les corresponde desempeñarse, lo cual supone, generar acciones a los fines de contribuir a revertir las situaciones problematizadoras actuales, propiciar la transformación de condiciones adversas al bienestar social, y por ende, la formación de ciudadanos capacitados para la vida.

Desde esta perspectiva, se hace oportuno revisar las estrategias que han contribuido con el desarrollo del conocimiento, en nuestro caso, específicamente el geográfico, someterlas al escrutinio de las demandas actuales, así como del avance de la ciencia, la tecnología, y los principios vinculados con el asunto ambiental.

En este contexto, se hace necesario demandar a los encargados de la formación de docentes, reflexiones de manera crítica sobre la pertinencia del currículo impartido, incluyendo en este, las estrategias didácticas que suponen la planificación y ejecución de técnicas y actividades a los fines de favorecer en el estudiante el desarrollo de las competencias profesionales, vinculadas con las políticas en materia educativa, las tendencias nacionales e internacionales, y las condiciones del contexto en el cual le corresponderá desempeñarse.

En este sentido, se asumió como intención teleológica para la presente investigación: develar con base en las experiencias-vivencias de los estudiantes del profesorado de Geografía e Historia de la Universidad Pedagógica Experimental Libertador en su Instituto Pedagógico de Caracas, los aportes del trabajo de campo en su formación como profesionales de la enseñanza de la Geografía. De allí que, el propósito de esta investigación apuntó hacia algo concreto, trascender lo que hasta el presente se ha entendido como una estrategia de enseñanza de la geografía y mostrar evidencias que permitan caracterizarla como una vía metodológica para la construcción social y totalística de los saberes.

Enmarcado en tal premisa, la investigación se desarrolló desde la perspectiva subjetivista, interpretativa $\mathrm{y}$ fenomenológica, con un diseño de campotranseccional-unieventual, cuyas aproximaciones teoréticas emergen a partir de los significados propios de las intersubjetividades de los estudiantes, como actores fundamentales en el proceso de aprendizaje.

Ahora bien, para efectos de presentar el producto investigativo, se seleccionó como texto académico el formato de los artículos científicos, por lo que la información se presenta estructurada en los siguientes apartados: (a) el trabajo de campo en la construcción del habitus geográfico, en el cual se presenta la disertación sobre las tendencias en materia de formación docente 
en geografía, así como el estado del arte de los estudios sobre la importancia del trabajo de campo, (b) métodos, en donde se arguyen asuntos vinculados al paradigma, tipo, nivel y diseño de la investigación, así como las etapas del método que oriento su ejecución, y los criterios para la selección de los informantes, (c) análisis y discusión de los hallazgos, a los fines de interpretar las categorías individuales-sintetizadas que emergieron producto de la sistematización de las experiencias de los informantes, aunado a la triangulación con referentes teóricos, y (d) conclusiones, a modo de cierre provisorio, desde el reconocimiento que los fenómenos sociales, son por esencia dinámicos $\mathrm{y}$ complejos, por lo que una investigación desde esta perspectiva no se considera acabada, sino más bien, se pretende develar su manifestación en un contexto y tiempo específico.

\section{El trabajo de campo en la construcción del habitus geográfico: una aproximación al estado del arte}

Las investigaciones enmarcadas en el abordaje de la formación docente, suelen atender, al menos dos aristas fundamentales: la generación de estrategias que favorezcan procesos de construcción de saberes, cada vez más eficientes, y, la profesionalización de educadores con competencias generales $\mathrm{y}$ específicas, enmarcados en perfiles de egreso, vinculados con las demandas de la sociedad y las tendencias mundiales.

En atención a esta última arista o línea de investigación, es oportuno referir que de acuerdo con lo planteado por Camargo et ál. (2004), la discusión en materia de formación docente, se vincula "con diferentes problemáticas, entre las que cabe resaltar por su ocurrencia, las siguientes: la calidad de la educación, la profesionalización del docente, los cambios que requieren las instituciones escolares y la práctica pedagógica que orienta las demandas" (p. 82).

Desde esta perspectiva, se ha venido reconociendo que "la formación docente es fundamental para la transformación de la sociedad que valora el desarrollo humano y los proyectos de vida de las personas en las que los diferentes procesos pedagógicos se convierten en una búsqueda permanente del ser y deber ser de la cultura de los sujetos de desarrollo" (Nieva y Martínez, 2016, p.18).

No obstante, en opinión de Miller (2002), los profesionales de la docencia, en ocasiones, evidencian una formación alejada de la creciente complejidad en la ocurrencia y abordaje de los diversos temas sociales y culturales que afrontan las escuelas.

De acuerdo con Morín (2007), lo anterior constituye una consecuencia de la supremacía de un conocimiento fragmentado, según las disciplinas, impidiendo a menudo operar el vínculo entre las partes y las totalidades, por lo que debe dar paso a un modo de conocimiento capaz de aprehender los objetos en sus contextos, sus complejidades y sus conjuntos.

Por otra parte, UNESCO (2009), ha recomendado a los responsables de la profesionalización de educadores, ampliar la formación "con planes y programas de estudios que den a los docentes la capacidad de dotar a sus alumnos de los conocimientos y las competencias que necesitan en el siglo XXI" (p. 3).

Por lo que en los últimos tiempos, se ha asumido como vía válida para la formación de docentes, los modelos por competencias, ya que permiten aproximarse a realidades complejas, desde la selección y/o diseño de estrategias didácticas que propicien en los estudiantes la motivación hacia el dominio de las herramientas necesarias que les permitan hacer frente a las exigencias de la vida cotidiana y profesional, así como sus 
contradicciones. Esto supone, centrar la atención en el desempeño humano integral, lo que implica la articulación del conocer con el plano del hacer y del ser (Tobón, 2006 y Roegiers, 2016).

De acuerdo con Aguiar y Rodríguez (2018), se debe "entender que ser competente no radica en que se posea algunas o muchas capacidades, pues la competencia no reside en los recursos (capacidades), sino en la movilización misma de los recursos; no es poseer, es utilizar" (p.144).

En palabras de Zabala (2006), se trata de considerar en los programas de formación docente las determinaciones legales, los contenidos básicos de nuestra disciplina (áreas de conocimiento o materias), el marco curricular al cual se circunscribe disciplina, nuestra propia visión de la disciplina y de su didáctica, así como las características de nuestros alumnos y los recursos disponibles.

En el caso específico de la formación de docentes especializados en la enseñanza de la Geografía, Marrón (2012), advirtió sobre:

la necesidad de enseñar y aprender Geografía con una metodología innovadora, acorde con las necesidades educativas de los ciudadanos del siglo XXI, capaz de aportar una visión amplia y enriquecedora de lo que constituye su objeto de estudio: la realidad socioespacial en sus múltiples escalas y vertientes. Esto supone la necesidad de que el profesorado esté abierto a las distintas corrientes científicas que configuran el panorama actual de la ciencia geográfica y de su didáctica, para evitar caer en visiones reduccionistas y mutiladoras de la realidad. (p. 324).

En este sentido, es oportuno referir a Jerez (2010), quien afirmó que en la actualidad la enseñanza de lo geográfico debe orientarse a responder cuestiones cada vez más complejas, demandante de profesionales conscientes de su rol social, reflexivos y capacitados para actuar, a fin de motivar a sus estudiantes en el abordaje de las relaciones entre las escalas global y local, los problemas ambientales como la sobreexplotación de los recursos, la degradación, alteración y contaminación de los paisajes, así como, los problemas sociales, entre los más urgentes y evidentes por su trascendencia global y local: el insostenible modelo de desarrollo, la pobreza, la crisis económica, ecológica o alimentaria.

De acuerdo con Moreno y Celis (2018), "es pertinente y relevante pensar y apostar por la construcción de una alfabetización espacial, entendida, en primera instancia, como el estudio y aprehensión del espacio de vida en correlación con el reconocimiento del ciudadano y de la ciudadanía como gestora de unos nuevos espacios y sociedades por medio de la cotidianidad" (p. 63).

En este sentido, Godoy (2006), expresó que la implementación del trabajo de campo, como estrategia didáctica en la formación del estudiante-docente, permite crear en estos un interés significativo hacia el conocimiento global del planeta, de lo ambiental, así como de su área de acción, propiciando la investigación científica de situaciones reales de la comunidad.

Así mismo, Lara (2011), afirmó con base en una investigación cualitativa, fundamentada en el método comparativo constante, que las vivencias del trabajo de campo tienen implicaciones pedagógicas al constituirse en una estrategia de enseñanza y de aprendizaje, ya que requieren de un plan de acción conformado por una secuencia de actividades planificadas y comprometidas en torno a un objetivo, favoreciendo un aprendizaje significativo donde se reflejan los cuatro pilares de la educación: conocer, ser, hacer y convivir. 
Por su parte, Delgado y Comino (2012), sostienen que el trabajo de campo, constituye una estrategia promotora de la formación por competencias geográficas para los estudiantes, instaurándose a su vez como una metodología de aprendizaje activa, altamente motivante, que fomenta el aprendizaje significativo, propiciando la reflexión sobre los conocimientos adquiridos, contextualizándolos y generando nuevos saberes basados en los ya existentes, y representa un ámbito propicio para introducir acciones de consolidación de competencias y destrezas útiles para el conocimiento del territorio: elaboración de mapas, análisis del paisaje, inventarios, entre otros. También refieren los autores que esta estrategia fomenta la concienciación social del alumnado, con respecto a realidades que hay que conocer y modificar, pudiendo propiciar un espíritu crítico y activo.

Aichino et al. (2013), con base en las reflexiones de profesores, estudiantes y egresados en geografía, en relación con la importancia del trabajo de campo, afirman que es el mismo campo quien sitúa al investigador para realizar situaciones de campo, porque las decisiones del investigador no nadan en un vacío, ellas están atravesadas por relaciones de poder que lo producen. Desde esta perspectiva, se puede afirmar que el contacto con la realidad y sus modos característicos, orientan e incluso jerarquizan la forma como el estudiante se aproxima a esta, atendiendo no solo a sus condiciones elementales, sino incluso a las complejas interacciones que se producen entre los elementos que la constituyen.

Di Franco y Maris (2017), refieren que las salidas de campo en geografía, propician la posibilidad de articular saberes, asignaturas, vínculos con la realidad y con el sentido de la formación, todos aspectos que fortalecen la comprensión y la transferencia.
Adicionalmente Núñez (2018), afirmó que durante el compartir propio de la actividad de campo, suelen emerger situaciones que dejan en evidencia los valores ético-sociales y ético-ambientales que sirven como referentes a los actores participantes de la actividad, por lo que el trabajo de campo como un método, fomenta el empoderamiento de valores pedagógicos, vinculados con el impulso que motiva a los estudiantes para comprometerse con su formación como docentes, aunado a la valoración positiva de lo aprendido, con lo cual se establecen patrones de referencia que tributan hacia el desarrollo profesional.

Moreno, Zagalaz y Elzel (2018), con base en una investigación cuantitativa, refieren que los estudiantes-futuros docentes, a partir de las experiencias en campo se sitúan en una actitud crítica en relación con el entorno, y desarrollan valores que contribuyen a los aprendizajes significativos.

Según Núñez (2019), la labor de campo, constituye una de las estrategias que por excelencia, contribuye con la formación de docente en el marco de la cotidianidad, afirmando entre otros asuntos:

El abordaje directo de la realidad estimula en los estudiantes el accionar de una pluralidad de procesos cognitivos, a partir de los cuales son capaces de percibir, codificar, analizar, interpretar, comprender y explicar las complejas interacciones que caracterizan los fenómenos estudiados en campo y proponer alternativas de solución que permitan mitigar o reducir los escenarios que están afectando el equilibrio socio-natural y, en consecuencia, la calidad de vida. ( $\mathrm{p}$. $56)$.

A modo de síntesis se puede afirmar que los autores referidos coinciden en expresar que el trabajo de campo, es en esencia una 
estrategia didáctica que favorece la formación integral del estudiante de geografía, toda vez que reduce las parcelas epistémicas entre la geografía física y humana, para transcender desde el contacto directo con los modos que caracterizan la realidad, a una comprensión compleja de los múltiples factores que intervienen en su configuración.

\section{MÉTODO}

Por la naturaleza ontológica del evento en estudio, se asumió como postura paradigmática la visión subjetivista, socioconstructivista o Interpretativista (Pérez Serrano, 1998), considerando que los hallazgos que constituyen la teoría, emerge como una construcción social, resultante de la sistematización de las experiencias y vivencias de los actores clave. En este sentido, se seleccionó el método fenomenológico, por constituir "una manera de describir la realidad o un método para abordarla e interpretarla" (Núñez, 2012, p. 20).

En este sentido, el investigador coloca dentro de paréntesis sus precogniciones y experiencias, para explorar desde la praxis de los informantes en primera instancia: ¿Cómo se concibe el trabajo de campo en su formación docente? ¿Qué aplicabilidad e importancia tiene lo vivenciado? ¿Es útil replicar la estrategia y lo aprendido en su ejercicio profesional?

Desde esta perspectiva, se asume el método fenomenológico propuesto por Husserl (1900, 1913, 1929 y 1970), considerando:

1. Constituye un nuevo método descriptivo, cuya aparición en el campo de la filosofía a principios del siglo $\mathrm{XX}$, lo orientó a subministrar el órgano fundamental de una filosofía rigurosamente científica y posibilitar, en un desarrollo consecuente, una reforma metódica de todas las ciencias (Husserl, 1900, p. 59).
2. Emerge como metodología alineada al estudio de las realidades sociales como son en sí, por lo cual se permite que éstas se manifiesten por sí mismas sin constreñir sus estructuras desde afuera, sino respetándolas en su totalidad (Martínez, 2009, p.167).

3. Describe el significado de las experiencias a partir de la perspectiva de quienes la han tenido (Zichi y Omery, 1994, p. 173).

4. El investigador debe procurar alcanzar la epoje o reducir sus creencias, valores, experiencias y emociones a la mínima expresión, a los fines de aproximarse al objeto de estudio sin prejuicios, sin intención de transformarle o intervenirle, sino con el claro propósito de conocerlo para describirlo. (Núñez, 2012, p.16).

\section{Informantes}

Para efectos de la presente investigación, se consideró como informantes a los actores clave del proceso de aprendizaje: los estudiantes del Profesorado de Geografía e Historia de la Universidad Pedagógica Experimental Libertador en su Instituto Pedagógico de Caracas.

La selección de estos, en primera instancia obedece a un criterio intencional, ya que se consideraron como tal, aquellos estudiantes que tuviesen experiencia de haber participado en por lo menos un trabajo de campo en el área de Geografía, y cuyas actividades fuesen ejecutadas en zonas alejadas a la ciudad de Caracas, permitiéndoles convivir por más de 2 días. Tales criterios obedecen a la intencionalidad del investigador de conocer las experienciasvivencias de los estudiantes-docentes en su proceso de construcción de aprendizajes, enmarcados en un contexto distinto al recinto universitario y su periferia, aunado a condiciones del compartir entre pares en situaciones propias de la cotidianidad. 
De allí que se interpretó la experiencia y significado atribuidos por los once estudiantes que voluntariamente acudieron a la convocatoria, interesados en compartir sus experiencias en participación en trabajos de campo.

\section{Etapas fenomenológicas Procedimientos de la investigación} $y$

Con el propósito de garantizar la sistematicidad durante el proceso investigativo, así como la construcción de la aproximación teórica correspondiente al evento en estudio, se asumió como momentos de la investigación, las etapas referidas por Husserl (1900, 1913, 1929 y 1970):

1. Etapa previa o de clarificación de los presupuestos, sustentada en los preceptos de Husserl (1970), tiene como propósito identificar entre los supuestos iníciales relacionados con el evento en estudio, los valores, actitudes $\mathrm{y}$ precogniciones, intereses, conjeturas, del investigador sobre el fenómeno que pretende abordar. Estos deberán hacerse evidentes como puntos de inicio $y$ precisar su posible influencia en la investigación, en otras palabras, es aquí cuando el investigador deberá alcanzar la epojé o colocar dentro de paréntesis sus conocimientos sobre el fenómeno, a fin de evitar su inclusión como parte de los hallazgos de la investigación. (Núñez, 2012, 2018 y 2019).

2. Etapa descriptiva: Sustentado en Husserl (1970), este momento tiene como propósito sine qua non lograr una descripción del objeto o fenómeno en estudio, que resulte lo más completa y no prejuiciada posible y al mismo tiempo, refleje la realidad vivida por cada sujeto, su mundo y su situación, en la forma más auténtica y válida (Núñez, 2012, 2018 y
2019). Es en esta etapa que se desarrolló la entrevista cualitativa a profundidad.

3. Etapa estructural: Tiene como fundamento los planteamientos de Husserl (1900 y 1929), y su eje central pretende orientar el estudio de las interpretación contenidas en los protocolos, para lo que se asumen ocho pasos, en los que conviene detenerse, de acuerdo con la prioridad temporal de la actividad en la que ponen énfasis (Núñez, 2012 y 2019):

a) Primer paso: Lectura general de la descripción de cada protocolo o transcripción de las entrevistas, a fin de obtener una visión de conjunto para lograr una idea general del contenido presente en los protocolos.

b) Segundo paso: Delimitación de las Unidades temáticas, basado en el planteamiento teórico desarrollado por Heidegger he incorporado por Martínez (2009). En este paso se debe "pensar -meditando- sobre el posible significado que pudiera tener una parte para el todo" (Martínez, 2009. p. 177). Se trata en todo caso de dar "denominación" a las expresiones de sentido, emergentes a partir del testimonio de los informantes.

c) Tercer paso: Con base en lo planteado por Leal (2001), en este paso se procura la determinación del tema esencial dominante en cada unidad temática, con miras a eliminar las repeticiones $y$ redundancias de estas últimas, $\mathrm{y}$, aclarar y elaborar su significado, lo cual se logra relacionándolas una con otra, desde la perspectiva del sentido del todo.

d) Cuarto paso: Expresión del tema central en lenguaje científico, aquí el "investigador reflexiona sobre los temas centrales a los que ha 
reducido las unidades temáticas (...) y expresa su contenido en un lenguaje técnico o científico apropiado". (Martínez, 2009, p.178).

e) Quinto paso: Integración de todos los temas centrales en una estructura particular descriptiva, es quizás uno de los más fundamentales. Martínez (2009) lo denomina "el corazón de la investigación y de la ciencia ya que durante el mismo se debe describir la estructura o las estructuras básicas de relaciones del fenómeno investigado" (p.178). Esto coincide con la determinación de las categorías fenomenológicas esenciales/individuales sintetizadas, según el planteamiento de Leal (2001)

f) Sexto paso: Integración de todas las estructuras particulares en una estructura general, cuya finalidad "es integrar en una sola descripción, lo más exhaustiva posible, la riqueza de contenido de las estructuras identificadas en los diferentes protocolos" (Martínez, 2009, p.181). Esto coincide con la determinación de las categorías fenomenológicas esenciales/universales, según el planteamiento de Leal (2001)

g) Séptimo paso: Entrevista final con los sujetos estudiados, cuyo propósito es darles a conocer los resultados de la investigación y oír su parecer o sus reacciones ante los mismos.

h) Octavo paso: Con base en lo planteado por Núñez (2019), incorporamos como un aporte al método fenomenológico, como último paso en la etapa estructural, la triangulación en la fase de interpretación, considerando en un franco diálogo de saberes: (a) la experiencia-vivencia de los docentes, (b) las proposiciones teóricas de los autores especialistas y (c) la experiencia-vivencia del investigador.

4. Discusión de los hallazgos: Fundamentada en los postulados de
Husserl (1913), tiene como objeto "relacionar los resultados obtenidos en la investigación con las conclusiones de otros investigadores para compararlas, contraponerlas, entender mejor las posibles diferencias $\mathrm{y}$, de este modo, llegar a una integración mayor y a un enriquecimiento del "cuerpo de conocimientos" del área estudiada" (Martínez, 2009, p.183).

\section{RESULTADOS}

\section{Hallazgos}

No se pretende cuantificar las contribuciones generadas por el trabajo de campo a nuestros estudiantes-docentes del Profesorado de Geografía e Historia, sino más, bien interpretar con base en las experiencias reportadas por estos, sobre algunos aportes a su formación profesional, asumiendo que la misión de la educación, es acuerdo con lo señalado por Morín, Roger y Motta (2003), "fortalecer las condiciones de posibilidad de la emergencia de una sociedad-mundo compuesta por los ciudadanos protagonistas, consciente y críticamente comprometidos en la construcción de una civilización planetaria" (p.122).

En este sentido, se distinguen como principales categorías fenomenológicas esenciales/individuales sintetizadas, emergentes de la interpretación de la experiencia de los estudiantes en el trabajo de campo: (a) construcción de la identidad docente, (b) desarrollo de la personalidad, (c) estrategia pedagógica, y (c) utilidad de lo aprendido.

\section{Aportes a la construcción de la identidad docente}

Entre los temas esenciales que sintetizan los aportes del trabajo de campo a favor de la 
construcción de la identidad profesional de los estudiantes del profesorado en Geografía e Historia, emergieron: (a) valoración del profesor de Geografía en la Sociedad; (b) cambios en la proyección personal y como docente, y (c) la configuración de la identidad profesional.

En lo concerniente a la valoración del profesor de Geografía en la Sociedad, el Informante 1, nos comentó a partir de la experiencia de campo:

uno empieza a comprender hacia dónde vamos como venezolanos y en cierta manera como los docentes y en especial los de geografía, tenemos la responsabilidad de hacer ver la importancia que se debe dar a la planificación y gestión del espacio, para que sea un ganar-ganar entre el hombre y la naturaleza, es lo que yo puedo observar en general de las paradas.

Se devela de esta expresión de sentido que el estudiante comprende su función como profesional de la enseñanza de la Geografía, transcendiendo los límites del "dador" de clase o "presentador" de información", para convertirse en promotor social de los cambios en materia de "planificación" y uso del espacio, tributando orientaciones en la localidad en dónde le corresponderá desempeñarse, desde una postura del "ganarganar", centrada en el equilibrio en la relación ser humano-naturaleza.

Lo anterior, promueve reflexiones críticas que propician en el estudiante cambios en su proyección personal y como docente, considerando los testimonios referidos por los informantes 2 y 3 , al expresar:

en mi caso, que además soy policía, veo que a futuro, a pesar de que a veces quisiera no continuar, pero saber que estoy aprendiendo cosas sobre mi país y que después las voy a enseñar, no me permiten abandonar. (Informante 2)

cuando me toque dar clase, será de una manera distinta, la geografía no va ser pura teoría, ya que eso la hace aburrida, no le da la práctica, no se evidencia lo que ella se merece. (Informante 3).

Como se puede apreciar, los estudiantesdocentes, coinciden en manifestar que la experiencia de campo les brindo una nueva manera de percibir los temas vinculados con la Geografía, superando el plano teóricomemorístico, y motivándolos a conocer más sobre el territorio venezolano, continuar su formación profesional y comprometerse con una enseñanza innovadora de los contenidos vinculados con esta área del saber.

En efecto, tal como lo plantea el Informante 1 , todo ello contribuye con la construcción de la identidad profesional:

luego del trabajo de campo tú dices, si vale la pena, esta carrera si vale la pena, enseñar lo que estamos nosotros aprendiendo, porque es para el beneficio tanto como de nosotros ,como de todas las demás personas, el poder reconocer los recursos con los que contamos, como nuestras acciones inciden sobre los otros componentes del ambiente, como podemos planificar el uso del territorio para generar bienestar, ese el valor que le da nivel profesional, como docente, como la profesión de la docencia, poder impulsar a la ciudadanía a conocer sobre estos temas.

Se devela de la expresión de sentido referida por el Informante 1, que la experiencia de abordar los temas en campo, permite conocer desde una dimensión 
práctica, los contenidos que suelen ser asumidos desde el plano teórico, lo cual genera una revaloración de los mismos, y por ende, favorece en el estudiante la autorreflexión para descubrir su del rol como docente de Geografía para en la sociedad, identificándose a su vez de manera vivencial con el perfil profesional.

En este orden de ideas, es propicio referir lo expuesto por Mórtola (2006), quien refirió que la identidad del docente es "la noción de sí mismo que porta un sujeto en cuanto enseñante", agregando que "expresa también las representaciones que el sujeto sostiene respecto del trabajo de enseñar, se construye fundamentalmente en la interacción" (p.86)

Tal "identidad no surge automáticamente como resultado de un título profesional, (...) es preciso construirla. Y esto requiere de un proceso individual y colectivo de naturaleza compleja y dinámica lo que lleva a la configuración de representaciones subjetivas" (Vaillant, 2007, p.3)

En el contexto universitario, de acuerdo con Sayago, Chacón y Rojas, (2008) la construcción de la referida identidad por parte de estudiantes en período de formación, "está fuertemente arraigada a las rutinas de quiénes fueron sus profesores en los otros niveles del sistema educativo, a las vivencias más significativas construidas con la familia, durante la infancia y al contacto directo con el contexto escolar" (p. 554).

En el caso particular de la formación del docente en geografía Herrero (2001), advirtió sobre la necesidad de formar al futuro profesor de geografía, de manera sintética y global, teniendo presente la interacción de los elementos naturales y lo humano, esto justificado en una la visión global basada en el componente espacial.

En este sentido, es oportuno referir lo expresado por Umaña (2004), quien afirmó que el trabajo de campo, contribuye con la formación geográfica, ya que permite al estudiante comprender la organización integral del espacio mediante un enfoque sistémico y lograr un cambio de visión de la realidad, la cual es interdependiente, dinámica, desordenada y multicausal, situación que ayuda a comprender mejor los lugares visitados.

De allí que durante el proceso de construcción de la identidad docente, el trabajo de campo aun cuando generalmente es planificado y ejecutado en el contexto de los contenidos propios de una unidad curricular específica, tal como lo refieren los informantes clave de esta investigación, contribuye con la generación de una revaloración de la visión del rol docente en la sociedad, así como de la proyección personal y profesional, desde una visión positiva de la carrera, apegada a su trascendencia social.

\section{Aportes al desarrollo de la personalidad}

Emergen como parte de la presente categoría fenomenológica individual/ sintetizada, los temas esenciales, que develan como la experiencia de campo, propicia el desarrollo de la personalidad, atendiendo fundamentalmente a: (a) la autoconfianza, y (b) el crecimiento personal.

En lo concerniente al primero de los temas esenciales referidos, los informantes 2 y 4, comentaron:

tú tienes una presión, la presión es por aprender, por desenvolverte y saber que cuando te vas a parar a hacer una discusión, tienes que emplear los mejores términos, los que aprendiste en clase, para desenvolverte bien $y$ hacer que la gente te entienda, osea $y$ el problema mío es que me pongo un poquito asustado cuando me toca desenvolverme de manera verbal, delante de un muchas personas, siento que me acople rápidamente al 
ejercicio de lo que se requieren en campo (Informante 2).

pese a que te hacen comentarios de lo bueno que pueda ser la estrategia, las experiencias varían y uno se hace una serie de interrogantes como ¿Qué va a pasar?, ¿Qué es esto?, entonces claro es la primera experiencia que tengo de trabajo de campo, ahora puedo decir que es agotador, llegar, estar en los sitios, subir, bajar, por mi edad, tengo 38 años pero van muchachos de $23 y$ 24 años, y yo dije nada me van a dejar botada y yo viendo como hago, porque yo vine a aprender, vine a estudiar $y$ yo me les pegaba atrás, pero fue agotador, el primer día yo decía: ¡Dios mío! ¿qué hago yo aquí? Porque claro, el calor, el caminar todo el día, luego en la noche llegar a discutir, en verdad que fue algo Diooos!! Pero después que pasó la discusión, y uno se toma un momento y descansas, uno empieza a rebobinar $y$ dice ime gusto! (Informante 4).

Se reconoce de las expresiones de sentido anteriormente referidas que antes del inicio de las actividades de campo, tenían muchas expectativas sobre su desempeño durante las jornadas, lo cual se justifica, ya que para algunos de ellos, tal experiencia, constituye la primera salida. Ante tal situación, este tipo de estrategias resulta "nueva", surgiendo dudas sobre ¿qué va a pasar?, lo que contribuyo inicialmente a condiciones psicológicas de presión y miedo. No obstante, en el transitar de la estrategia, estos logran sentirse confiados, "acoplados" e incluso "les gusta" la manera como están construyendo los saberes.

El Informante 1, advirtió que la estrategia de campo, aporta al crecimiento personal, " $a$ partir de esta experiencia te das cuenta de tus potencialidades, osea, hizo como que, una línea en forma ascendente, hacia ser mejor personas y profesionales, cada día."
Es propicio destacar, de acuerdo con lo expresado por Seelbach (2012), que la personalidad es un elemento fundamental en la formación docente: "permite conocer la manera en la cual una persona puede aprender de su entorno" (p.9). Agregando que "se puede definir como la estructura dinámica que tiene un individuo en particular; se compone de características psicológicas, conductuales, emocionales y sociales" (p.9).

Desde esta perspectiva es válido referir a Digman (1997), quien afirmó que la reflexión en positivo de las experiencias, generalmente reflejan el crecimiento personal.

Tal afirmación, se sostiene en el llamado modelo de los cinco grandes de la personalidad (Laak, 1996 y Bigi, 2015), arguyendo que esta suele configurarse producto de la interacción entre: (a) Extroversión (Surgencia), afecto, gregarismo, asertividad, actividad, búsqueda de emociones, emociones positivas, (b) Agradabilidad (Simpatía), confianza, honradez, altruismo, cumplimiento, modestia, sensibilidad, (c) Consciencia (Conformidad, Seguridad), competencia, orden, obediencia, lucha por el logro, autodisciplina, reflexión, (d) Neuroticismo (Estabilidad Emocional, Inquietud), Ansiedad, hostilidad colérica, depresión, timidez, impulsividad, vulnerabilidad, y (e) Apertura a la experiencia (Cultura, Intelecto, "Intelectancia"), fantasía, estética, sentimientos, acciones, ideas, valores.

De acuerdo con Olivera y Simkin (2016), aquellos elementos valorados socialmente, entre estos: el éxito personal, la autonomía, o la capacidad establecer relaciones interpersonales satisfactorias, influyen de manera positiva en la percepción del bienestar, ya que los rasgos de personalidad contribuyen diferencialmente a alcanzar estas metas u objetivos sociales. 
Al respecto Núñez (2018), refirió que la participación en el trabajo de campo, fomentan valores políticosocial, tales como: respeto, empatía, integración, trabajo en equipo, compartir, amistad, sinergia, liderazgo, resolución de situaciones de conflicto, entre otros. Estos garantizan la participación afectiva de los estudiantes y el armonioso desarrollo de la estrategia en un sano convivir. Adicionalmente se consolidan valores individuales, tales como: el esfuerzo personal y la autoconfianza o seguridad en sí mismo, como elementos que tributan no solo hacia una participación efectiva, sino además, al desarrollo personal.

Es posible asumir que la valoración positiva de los estudiantes-docentes en atención a sus experiencias en el trabajo de campo, sintetizadas en aspectos vinculados a la autoconfianza y el desarrollo personal, posiblemente esté vinculado con la sensación de bienestar vinculada a la participación en las actividades, caracterizada por un alto sentido de extroversión, agradabilidad, conciencia y apertura a la experiencia, así como un reducido neuroticismo.

\section{El trabajo de campo como método para la construcción del conocimiento geográfico}

Se develan en este apartado algunas apreciaciones de los estudiantes-docentes sobre: (a) la importancia del trabajo de campo como un método para la construcción de los saberes, (b) importancia de aplicación de la estrategia en los distintos niveles educativos, (c) réplica de la estrategia en los contextos escolares, y (d) su valoración.

Siguiendo el orden de idea, se destaca como importancia de la estrategia para la construcción de los saberes, lo referido por los informantes 1, 3 y 5, quienes coinciden en expresar que el trabajo de campo, fomenta el aprendizaje significativo ya que: el trabajo de campo primeramente es una experiencia significativa, en la cual mezcla la teoría con la práctica, en cierta forma se puede asimilar más rápido, ya que uno va aprendiendo con el trascurso de lo que sería el trabajo de campo, adicionalmente lo vas palpando y se hace como que más divertido de cierta forma el aprendizaje. (Informante 1).

es fascinante, por todo lo que ocurría allí, por ese pasado-presente que está allí y que podemos verlo, que es historia y al mismo tiempo historia geológica por las evidencias que están allí. (Informante 3).

la experiencia en este trabajo de campo fue súper significativa, porque yo personalmente de verdad, pude palpar los contenidos que vimos en la teoría. (Informante 5).

Tales expresiones de sentido, revelan que la estrategia resulta importante para el proceso de construcción de los saberes geográficos en los estudiantes, toda vez que: (a) fomenta el aprendizaje significativo, (b) vincula teoría-práctica, (c) facilita la compresión de los contenidos, y (d) promueve experiencias de aprendizaje positivas o "divertidas".

Siendo así, la importancia de su aplicación en distintos niveles educativos, constituye un elemento relevante para la selección y diseño de estrategias didácticas, de acuerdo con lo expresado por los informantes 1, 4, 6 y 7, esta permite:

mostrarle el sector donde ellos se encuentran, como es su dinámica, como sería el abordar de cierta forma el estudio geográfico de ese sector, explicarle cual es la importancia también que tiene, que brinda estudiar el terreno, estudiar el ambiente (...) como también nosotros podemos 
aportar soluciones, osea hay varios proyectos que se han realizado, lo bueno uno pudiera demostrárselos a ellos y con diferentes estrategias también en el aula de clases, que bueno te pueden ayudar también a lo que ellos ven, pueden tener esa sensibilidad por los temas ambientales (Informante 1).

yo sí creo, que es una estrategia que debe ser aplicada, es más debería hacer obligatoria, como usted siempre nos dice: ¡la geografía entra por los pies!, eso es verídico, no hay mejor manera de comprender la geografía que a través del trabajo de campo, y si es así, y queremos enseñar geografía, debemos hacer uso de esta estrategia (Informante 4).

todo esos contenidos que uno ve en la materia y que es provechoso para uno, para ayudar a los estudiantes a comprender su realidad, el trabajo de campo seguramente, al igual que ocurre con nosotros en la Universidad, les facilitará la comprensión a nuestros estudiantes cuando estén estudiando ese tipo de contenidos (Informante 6).

en la fase de ensayo pude dar clase en el liceo André Bello, di Geografía y pienso que en algún momento puedo hacer un trabajo de campo, no en esta magnitud, pero si procuraría la forma de sensibilizar a la Dirección del plantel, para hacer un trabajo de campo, puede ser en la parte de geografía si se puede decir, aquí mismo tenemos el cerro el Ávila, podemos dar una clase en donde abordemos la dinámica de la ciudad de Caracas, pudiéramos ir al Ávila para no ir tan lejos, siento que es importante y salir de la monotonía del aula, a las calle, a la vivencia, a que ellos reconozcan, que sí que existe una altura significante aquí en Caracas, el
Ávila, que representa el Ávila para nosotros en Caracas, que divide el Ávila y el litoral de Vargas (Informante 7).

Como evidencia de la factibilidad de replicar la estrategia en el nivel de educación básica y media diversificada, los informantes 1 y 4 , refieren que:

yo después del trabajo de campo, fui con mis estudiantes, ahora que tengo la oportunidad de trabajar en el área, con un cartograma del sector, fue un recorrido por la zona de Antimano, para observar cuales son las zonas de alto riesgo, para que ellos identificaran zonas de peligro, también zonas en donde puedan refugiarse ante cualquier fenómeno, eso de cierta forma motivo a los chicos(...) si se pueden hacer recorridos en las zonas próximas a las instituciones (Informante 1).

el lunes les mostré las fotos y les iba explicando, y ellos claro, decían iprofesora llévanos!, yo les digo, ya va, eso requiere de una organización, mi profesor tiene todo el semestre planificando la actividad, eso no es así, quizás cuando estén en quinto podamos hacerlo, porque ellos están en cuarto, pero podemos ir al Ávila que es lo más cercano y allí trabajamos un poco las rocas, tal vez la vegetación, los pisos térmicos y los cambios de temperatura, la dinámica espacial de Caracas, algo siempre se puede hacer. (Informante 4).

Los estudiantes valoran positivamente la estrategia como parte de su proceso de formación como docentes, en este sentido, los informantes 4 y 7 expresaron:

Muchos creen que es un paseo o algo así y no, es un trabajo serio, académico en donde se va a aprender trabajando o a construir el conocimiento. (Informante 4). 
4 días para mí fue una experiencia única y de verdad que para mí significo mucho, a mi familia, a mis amigos del trabajo les comente, incluso las fotos, le mostré mira ven a ver la foto mira aquí estuvimos en Araya, tengo un conocimiento más amplio de lo que es la costa oriental, pero de si el trabajo de campo muy significativo (...), para mi es importante y quisiera contribuir con mi experiencia, aportarla o que mi experiencia se oiga para que no solamente Geografía Física si no varias materias pudieran hacer este tipo de actividades que es muy importante es significante para mí (Informante 7).

De lo anteriormente referido, se evidencia la alta valoración que hacen los estudiantes a la estrategia de campo, como medio para la aproximación, reconocimiento, análisis, interpretación e interrelación de los elementos constitutivos de las realidades en estudio. El referido abordaje vivencial, fomenta en estos la construcción de los saberes geográficos desde una perspectiva significativa.

La posibilidad de replicar durante su desarrollo profesional, la estrategia y lo aprendido, resulta de gran importancia dentro del proceso de formación profesional, ya que como lo planteó Chastain (1988) "las experiencias que tienen un resultado positivo tienden a ser repetidas y aquellas con resultados negativos a ser evitadas" (p. 167).

La sola posibilidad de que los estudiantes-docentes repliquen o consideren hacer un trabajo de campo con sus alumnos, constituye un indicador vivencial sobre lo positivo de la aplicación de la estrategia para los estudios geográficos, y por ende, contribuye al empoderamiento de un perfil profesional orientado hacia la construcción social del conocimiento desde la cotidianidad y el contexto local.

\section{Utilidad de lo aprendido para la vida profesional}

Constatar la utilidad de lo aprendido durante las actividades de campo, y sus contribuciones en el desempeño profesional, puede ser motivo de un debate interminable, sobre todo porque a la luz de esta disertación, la vida profesional de nuestros estudiantesdocentes apenas inicia, sin mayores experiencias en el ejercicio profesional, y es justamente durante ese recorrido como profesionales de la docencia, cuando emergerán las evidencia del cúmulo de conocimientos conceptuales, procedimentales $\mathrm{y}$ actitudinales, fomentados durante su transitar universitario y en particular durante las experiencias de trabajo de campo en su formación como docentes.

No obstante, con base en algunas reflexiones de nuestros estudiantes, se intenta de manera provisional, generar una aproximación al referido planteamiento, considerando que al respecto emergieron como temas esenciales constituyentes de la presente categoría fenomenológica esencialindividual sintetizada: (a) necesidad de aportar soluciones a los problemas detectados, (b) la transferencia de conocimiento, y (c) la relación escuelacomunidad.

En lo referente al asunto denominado: necesidad de aportar solución a los problemas detectados, los informantes advierten que el trabajo de campo, motivo en ellos las siguientes inquietudes:

de repente nuestro gobierno y nadie haga algo para que eso cambie, bueno, eso me genera cierta perturbación en mi carrera, porque ahora siento que quisiera dar clase en ese lugar, para trabajar con esas comunidades $y$ mostrarles que posiblemente también estén contaminando el mar, porque vi una parte contaminada. (Informante 1). 
por ejemplo si yo soy experta en Geografía y sigo aprendiendo como en el trabajo de campo, bueno yo voy a mejorar un poquito a la sociedad, aportándole de lo que yo sé, creo que puedo aportar como docente, pero también como especialista en Geografía, trabajando en la solución de los problemas de las comunidades. (Informante 3).

Creo que como profesor, debemos impulsar estudios hacia esas zonas y pedirle ayuda a las autoridades, hacerles llegar a las inquietudes que tenemos, son poblaciones que tienen mucho para dar, recordemos que son pueblos que practican actividades económicas que se explotan y la cantidad monetaria que se les aporta a esos pueblos, no es acorde a lo que ellos producen, entonces debemos hacer más estudios hacia esas zonas $e$ informar a las autoridades. (Informante 8).

Justamente cuando hablamos el último día en la reflexión, a parte de los conocimientos que iba tomando día a día, ese día fue el que más me marcó, pues, comencé a ver esa valoración por lo que tenemos, pero que no lo estamos aprovechando, veo que hay una desinformación completa en las aulas de clase, y yo doy clase, sobre el conocer a Venezuela, sobre la gestión de riesgo, y creo que como profesor debo divulgar esa información. (Informante 9).

Con base en las afirmaciones anteriores, se hace necesario resaltar:

independientemente que el trabajo de campo sobre el cual han sido consultado los informantes, se enmarca dentro de los saberes propios de la Geografía Física de Venezuela y en consecuencia, para tal fin han sido diseñadas cada una de las actividades, el desarrollo de la experiencia, favorece en los estudiantes-docentes, entrar en contacto con realidades socioeconómicas poco favorables para los habitantes de los sectores visitados, de allí que, (b) estos sientan la iniciativa como ciudadanos y profesionales en formación, de aportar desde sus cogniciones previas e incluso desde las construidas durante el trabajo de campo, posibles soluciones en el ámbito educativo y geográfico, a los fines de mitigar o revertir las condiciones actuales y mejore la calidad de vida de los lugareños.

Siguiendo el orden de ideas, emerge como tema esencial la trasferencia de conocimiento, sobre la base de las siguientes expresiones de los informantes 10 y 11, quienes comentaron:

en el estado Vargas podemos ver todo lo que son los acantilados, los que se formó después la de la tragedia de Vargas, se aplica eso para explicarle todo lo que ocurrió y por qué ocurrió la tragedia de Vargas. (Informante 10).

Entonces si uno ya lo ve, puede dar una mejor descripción de los lugares que ya visito, y creo que como formación, no solo porque está el concepto y uno lo vio aquí, pero ahora también está la experiencia y eso es un refuerzo, o sea el trabajo de campo es un refuerzo a lo que ya uno vio en clase, es un súper refuerzo, porque si habían cosas que se habían quedado allí con dudas, en el trabajo de campo se aclararon perfectamente y uno las agarró allí. (Informante 11).

Se devela con base en las expresiones anteriores, que la referida trasferencia de conocimiento, debe ser entendido al menos en dos direcciones: por un lado, el conocimiento construido durante el trabajo de campo, puede ser aplicado para el análisis de situaciones similares en otras unidades del paisaje, como a modo de ejemplo ha referido el informante 10, al comparar los acantilados 
estudiados en el oriente venezolano con los del litoral central en el estado Vargas, así como, la dinámica de las referidas vertientes; por otro lado, en el ámbito pedagógico, los informantes manifiestan a propósito de la experiencia vivida, que esta le ha dado la formación necesaria para abordar los problemas y fenómenos estudiados, en su posterior ejercicio profesional, trasladando a sus respectivos estudiantes los saberes construidos.

En relación con esto último, el Informante 1 destaca otra de las aplicaciones que tiene lo aprendido en la experiencia de campo, vinculándolo con las competencias que les brinda para abordar situaciones directas en sus espacios de acción, propiciando la relación escuela-comunidad, considerando:

tú le mandas a investigar en su casa, por ejemplo con lo del tema de la contaminación, que si ellos reciclan directamente en su casa como tal, se pudiera decir, no sé, ese valor de cuidar las cosas, ayudaría también a sensibilizar el hecho que bueno si en la misma escuela donde botan los desperdicios, si lo botan en la calle, si lo queman porque también queman la basura.(...) ellos deben entrar en contacto con la realidad, en el recorrido de su casa al colegio, es una manera de que observen y se sensibilicen por los impactos.(...) para tener una vivencia más cercana a la realidad, por eso hablo del recorrido en las zonas próximas al colegio como una alternativa.

La experiencia de campo no solo facilita el aprendizaje, favoreciendo su construcción colectiva, sino que en el caso específico de los estudiantes-docentes en ejercicio, les motiva y da herramientas para abordar situaciones problemáticas en sus respectivas comunidades, como por ejemplo refiere el
Informante 1, al ser consultado sobre el asunto:

en la comunidad en dónde queda mi escuela, la gente quema la basura y siempre eso me había llamado la atención pero uno lo comenta y ya, pero después del trabajo de campo me di cuenta que el profesor no solo da clase en el aula, sino que yo también puedo llevar a mis estudiantes a recorrer la comunidad y bueno les explico lo del relieve, lo de la vegetación que ha sido intervenida, pero también problemas como ese de la basura y el de la quema de esos desechos, para que ellos se den cuenta que eso no está bien, que eso genera contaminación, que deben propiciar en su casa que la basura la deben colocar en bolsas cerradas, que como comunidad debemos organizarnos y llamar a las autoridades para que recojan la basura, y bueno ahora también hablo con los representantes cada vez que nos reunimos.

En síntesis, el trabajo de campo propicia la construcción de unos saberes útiles para la vida profesional $\mathrm{y}$ personal de los estudiantes-docentes, toda vez que les empodera en: (a) el dominio en los planos conceptual, procedimental y actitudinal, (b) la transformación de sus cogniciones y visión de la realidad ambiental, y (c) les permite comprender las situaciones locales, reflexionar sobre sus potenciales causas y proponer alternativas para la prevención, mitigación y transformación de las realidades actuales, a fin de contribuir con el bienestar que conlleva a una calidad de vida en armonía con el equilibrio planetario.

Resulta positiva la valoración de los estudiantes-docentes sobre lo aprendido de manera significativa y profunda, resaltando la posibilidad de aportar soluciones a los 
problemas locales, transferir los conocimientos no solo a sus futuros estudiantes, sino incluso para el abordaje de situaciones y/o condiciones similares a las estudiadas en campo, así como, la posibilidad de abordar desde las escuela, los problemas ambientales presentes en la comunidad. Esto de acuerdo con Beas, et ál. (2001), implica "el dominio, la transformación y la utilización" que se haga de ese conocimiento para comprender y resolver problemas reales.

Tal valoración, de acuerdo con Herrero (2001), "servirán luego de punto de partida para sentar ciertas nociones básicas y fijar una terminología y, por vía de la comparación, estas nociones podrán ser extendidas a otros paisajes geográfico, físico y humano, fuera de la observación directa" (p.190).

\section{CONCLUSIONES}

De acuerdo con las experiencias-vivencias reportadas por los estudiantes del profesorado de Geografía e Historia de la Universidad Pedagógica Experimental Libertador en su Instituto Pedagógico de Caracas, los aportes del trabajo de campo en la su formación profesional, pueden ser sintetizados en las cinco dimensiones del conocimiento, a saber: ontológica, epistemológica, axiológica, metodológica y teleológica.

En relación con la dimensión ontológica, se devela que el trabajo de campo aproxima al estudiante-docente a las diversas condiciones intervinientes en la configuración de realidades específicas. De allí que en su formación, se transcienden los muros de la universidad, para ubicarlos en interacción directa con la complejidad del espacio geográfico, generando en estos una concepción multifactorial de los fenómenos identificados, cuyo abordaje, requiere integrar los diversos enfoques disciplinares aprendidos a lo largo de la prosecución de sus estudios profesionales.
En lo epistemológico, el trabajo de campo contribuye con la formación complementaria en las dimensiones teórica y práctica, toda vez le motiva a construir, deconstruir y reconstruir sus cogniciones en atención a las experiencias y evidenciadas vinculadas con los fenómenos abordados. De esta manera, se fomenta el aprendizaje significativo desde un enfoque socioconstructivista de los asuntos geográficos, ya que el estudiante participa de manera individual y colectiva en la construcción de los saberes, mediante la observación, análisis, contrastación, síntesis y transferencia de información.

En relación con la dimensión axiológica, el trabajo de campo fomenta la formación de profesionales con un perfil sensibilizado por los asuntos sociales, caracterizado por la valoración de su rol como promotor de cambios, mediador en la formulación de soluciones a las situaciones problemáticas detectada, sin descuidar su posición como orientador-facilitador de los procesos que conllevan a la construcción de sus propios saberes. En el plano personal, propicia el crecimiento personal fundamentado en la autoconfianza.

En relación con lo metodológico, la estrategia sitúa al futuro profesional de la docencia en contacto directo con las situaciones problematizadoras de la localidad. Por lo que motiva la formulación de posibles soluciones, la transferencia de conocimiento, así como los mecanismos para la integración escuela-comunidad.

En cuanto a la dimensión teleológica, se empodera al estudiante-docente de su perfil profesional específico, toda vez que lo vincula con el contexto en estudio, generando sensibilidad y sentido de identidad profesional, le proyecta en el abordaje didáctico de situaciones específicas de realidades particulares, como vía para promover ciudadanos ambientalmente 
responsables desde el accionar pedagógico, individual y colectivo.

A modo de aproximación teorética, se pude afirmar que el trabajo de campo, más que una estrategia didáctica, constituye un método para el abordaje de la realidad, permitiendo la integración teoría-practica desde una visión compleja, y promoviendo un perfil integral del estudiante del profesorado de Geografía, ya que como se expresó anteriormente: minimiza la fragmentación del conocimiento geográfico y su atomización epistémicas entre la geografía física y humana, para transcender hacia la complementariedad ontoepistemológica y metodológica, que permita comprender los fenómenos espaciales.

\section{REFERENCIAS}

Aguiar, X. y Rodríguez, L. (2018). La formación de competencias pedagógicas en los profesores universitarios. Edumecentro, 10(2), 141-159. Recuperado de http:// scielo.sld. cu/ pdf/edu/v10n2/edu 11218.pdf

Aichino, G., Arancibia, L., Astegiano, N., Asis, Y., Barrera, E., Cavanagh, E., Cisterna, C., González, D., Luna, L., Palladino, L., Padrazzani, C., Rodigou, J. Trabajo de campo y formación del geógrafo. Algunos aportes para su reflexión. Cardinalis. Revista del Departamento de Geografía, 1 (1), Recuperado de https://ri.conicet.gov.ar/handle/11336/ 24763

Beas, J.; Santa Cruz, J.; Thomsen, P., y Utreras, S. (2001). Enseñar a pensar para aprender mejor. Santiago: Ediciones Pontificia Universidad Católica de Chile

Bigi, M. (2015). El modelo de los cinco factores de la personalidad y la teoría triangular del amor. Revista de Investigación en Psicología Social, 1 (2), 68-77. Recuperado de https://publicaciones.sociales.uba.ar/ind ex.php/psicologiasocial/ article/download/1228/1118
Camargo, M., Calvo, G., Franco, M., Vergara, M., Londoño, S., Zapata, F. y Garavito, C. (2004). Las necesidades de formación permanente del docente. Educación y Educadores, 7, 79-112. Recuperado de https://dialnet.unirioja.es/descarga/arti culo/2041051.pdf

Chastain, K. (1988). Developing Second Language Skill. Theory and Practice. [Desarrollo de habilidades de segundo idioma. Teoría y práctica] San Diego: Harcourt Brace Jovanovich Publishers

Delgado, J. y Comino, J. (2012). El trabajo de campo y las competencias geográficas en el estímulo para el estudio de la geografía: aplicación en un aula de $2^{\circ}$ de bachillerato. Didáctica Geográfica, 13, 3556. Recuperado de http://www.agegeografia.es/didácticageografica/index.p hp/didacticageografica/article/viewFile/ $95 / 95$

Di Franco, M. y Maris, S. (2017). El Campo de la Práctica en Geografía. Una formación que trasciende el aula universitaria. Huellas, 21 (2). Recuperado de https://dialnet.unirioja.es/descarga/artic ulo/6206622.pdf. DOI: http://dx.doi.org/10. 19137/ huellas2017-2115

Digman, J. (1997). Higher-Order factors of the big-five. [Factores de orden superior de los cinco grandes] Journal of Personality and Social Psychology, 73(6), 1246-1256. Recuperado de https://doi.org/10.1037/00223514.73.6.1246. DOI: $10.1037 / / 0022-$ 3514.73.6.1246

Godoy, I. (2006). El trabajo de campo en la enseñanza de la Geografía. Sapiens. Revista Universitaria de Investigación, 8 (2), 137-147

Herrero, C. (2001). La docencia, uno de los oficios del Geógrafo. Boletín de la Asociación Española de Geografía, 31, 185-191. Recuperado de https://dialnet. unirioja.es/ descarga/articulo/1122 539.pdf

Husserl, E. (1900). Investigaciones lógicas. Madrid: Alianza.

Husserl, E. (1913). La idea de la fenomenología [Traducido por Jesús 
Adrián Escudero, 2012] Recuperado de http://www.casadellibro.com/libro-laidea-de-la-fenomeno logia/9788425428371/1961681

Husserl, E. (1929). Meditaciones cartesianas. Ciudad de México: Fondo de Cultura Económica

Husserl, E. (1970). Philosophie der Arithmetik. Mitergänzenden Texten [Filosofía de la aritmética. Textos suplementarios] (1890-1901), Londres: Den Haag, Martinus Nijhoff, Kluwer Academic Publisher

Jerez, O. (2010). Fundamentos geográficos y curriculares en la formación del profesorado. ¿Qué, dónde y cuándo enseñar? En M. Marrón y M. de Lázaro (eds.). Geografía, educación y formación del profesorado en el marco del Espacio Europeo de Educación Superior, Madrid: Grupo de Didáctica de la Geografía de la Asociación de Geógrafos Españoles y Departamento de Didáctica de las Ciencias Sociales de la Universidad Complutense de Madrid. 405-421. Recuperado de https://docplayer.es/5740 9186-Geografiaeducacion-y-formacion-del-profesorado-enel-marco-delespacio-europeo-deeducacion-superi or.html

Laak, J. (1996). Las cinco grandes dimensiones de la personalidad. Revista de Psicología de la PUCP, XIV (2), 129-181. Recuperado de https://dialnet. unirioja.es/descarga/

articulo/4625401.pdf; Texto

Lara, S. (2011). Las vivencias estudiantiles del trabajo de campo y sus implicaciones pedagógicas. Revista de investigación, 35 (73), 195-2018. Recuperado de https://www.redalyc.org/pdf/3761/376 140388009.pdf

Leal, N. (2001). El Método Fenomenológico: Principios, Momentos y Reducciones. Revista Electrónica de Investigación Científica, Humanística y Tecnológica, 1(5), Recuperado de http://revistadip.una.edu.ve/

Marrón, M. (2012). Educación geográfica y formación del profesorado. Desafíos y perspectivas en el nuevo Espacio Europeo de Educación Superior (EEES). Boletín de la asociación de Geógrafos españoles, 57, 313-341. Recuperado de https://dialnet.unirioja.es/descarga/artic ulo/3814337/1.pdf

Martínez, M. (2009). Comportamiento humano: nuevos métodos de investigación. Ciudad de México: Trillas

Miller, E. (2002). Políticas de formación docente en la mancomunidad del Caribe. En UNESCO/OREALC Formación docente: Un aporte para la discusión. La experiencia de algunos países. 15-34. Santiago, Chile: UNESCO

Moreno, G., Zagalaz, M. y Elzel, L. (2018). Las experiencias de trabajo de campo para aprender y enseñar geografía: El caso de la comunidad educativa de Osorno, Chile. Revista de Historia y Geografía. 38. 147159. Recuperado de https: //pdfs.semanticscholar.org/2589/4435a 34e2a5ca787d74be8d323bd4159dc32.p $\mathrm{df}$

Moreno, N. y Celis, A. (2018). Educación geográfica, formación docente y vida cotidiana. Bogotá: Universidad Pedagógica Nacional. Recuperado de http://repositorio.pedagogica.edu.co/bitstr eam/handle/20.500.12209/9394/CIUPLbro $\%$

203\%20final.pdf?sequence=1\&isAllowed=y

Morín, E., Roger E. y Motta R. (2003). Educar en la era planetaria, Barcelona: Gedisa.

Morín, E. (2007). Los siete saberes necesarios para la educación del futuro. Buenos Aires: Ediciones nueva visión

Mórtola, G. (2006). Una aproximación narrativa a la construcción de la identidad laboral docente: algunos aspectos biográficos previos a la formación inicial. Educación, Lenguaje y Sociedad, 4 (4). Recuperado en http://www.biblioteca.unlpam.edu. ar/pubpdf/ieles/n04a05morto la.pdf

Nieva, J. y Martínez, O. (2016). Una nueva mirada sobre la formación docente. Universidad y Sociedad, 8(4), 14-21. Recuperado de http://scielo.sld.cu/pdf /rus/v8n4/rus02 416.pdf 
Núñez, F. (2012). La fenomenología: epistemológica u ontológica, de Husserl a Heidegger. COHISREMI, 8(2), 14-23. Recuperado de https://www.research gate.net/publication/284188654_LA_FEN OMENOLOGIA_EPISTEMOLOGIC_U_ONTO LOGICA_DE_HUSSERL_A_HEIDEGGER

Núñez, F. (2018). Axiología del trabajo de campo en Geografía: Una teorización fenomenológica. Revista de Educación, 14, 25-47. Recuperado de https://fh.mdp. edu.ar/revistas/index.php/r_educ/article /view/2567 /2919

Núñez, F. (2019). Aportes del trabajo de campo a la construcción de los saberes geográficos: una teorización fenomenológica a partir de las experiencias-vivencias de los estudiantes. Revista de Investigación y Postgrado, 34(1), 37-58. Recuperado de https://dialnet.unirioja.es/descarga/artic ulo/6931374.pdf

Olivera, M. y Simkin, H. (2016). Factores de la personalidad y su relación con el bienestar subjetivo y psicológico. Hologramática, 25(1), 77-96. Recuperado de

http://www.cienciared.com.ar/ra/usr/3 /1669/holo25_v1_p77_96.pdf

Roegiers, X. (2016). Marco conceptual para la evaluación de competencias. UNESCO-OIE. Recuperado de http://www.ibe.unesco.org/sites/default /files/resources/ipr4-roegierscompetenciesasses sment_spa.pdf

Sayago, Z., Chacón, M. y Rojas, M. (2008). Construcción de la identidad profesional docente en estudiantes universitarios. Educere: Investigación arbitrada, 42. Recuperado en http://www.saber.ula.ve/bitstream/123 456789/26312/1/articulo1 5.pdf

Seelbach, G. (2012). Teoría de la personalidad. México: Red tercer milenio S.C. Recuperado de http://www.aliat.org.mx/BibliotecasDigitales /Psicologia/Teorias_de_la_personalidad.pdf
Tobón, S. (2006). Aspectos básicos de la formación basada en competencias. Talca: Proyecto Mesesup. Recuperado de https://maristas.org.mx/gestion/web/do ctos/ aspectos basicos_formacion_competencias.pdf

Umaña, G. (2004). Importancia de las salidas de campo en la enseñanza de la Geografía. Folios Segunda época, 20, 105-120, Recuperado de https://www. researchgate.net/publication/31937327 2_Importancia_de_las_salidas_de_cam po_ en_la_ensenanza_de_la_Geografia

UNESCO. (Julio, 2009). Conferencia Mundial sobre la Educación Superior: La nueva dinámica de la Educación Superior y la investigación para el cambio social y el desarrollo. París. Recuperado de http://unesdoc.unesco.org/images/0018 /00 1832/183277s.pdf

Vaillant, D. (Septiembre de 2007). La identidad Docente [Documento en línea]. Ponencia presentada en el I Congreso Internacional "Nuevas Tendencias en la Formación Permanente del Profesorado, Barcelona. Recuperado de http://www. ub.edu/obipd/docs/la_i dentidad_ docente_vaillant_d.pdf

Zabala, M. (2006). Guía para la planificación didáctica de la docencia universitaria en el marco del EEES. Documento policopiado. Santiago de Compostela: Universidad de Santiago de Compostela. Recuperado de https://www.udc.es/ grupos/ apumefyr/docs/guiadeguias.pdf

Zichi, M. y Omery, A (1994) Escuelas de fenomenología: implicaciones para la investigación. En J.M. Morse (Comp). Asuntos críticos en los métodos de investigación cualitativa (pp 160 -182). Antioquia: Editorial Universidad de Antioquia 